\title{
Smart sealing for magnetorheological fluid actuators
}

Journal of Intelligent Material System and Structures 2019, Vol. 30(5) 689-700 (C) The Author(s) 2018 Article reuse guidelines: sagepub.com/journals-permissions DOI: | 0.1 I77/I045389XI775426| journals.sagepub.com/home/jim (SAGE

\author{
Christian Hegger (iD and Jürgen Maas
}

\begin{abstract}
In automotive and industrial environments, magnetorheological fluid-based applications such as clutches and brakes are gaining continuously more attention. However, one of the serious challenges for their application are drag losses at high shear rates. While viscous losses of the magnetorheological fluid can be eliminated by a magnetorheological fluid movement control based on partially filled shear gaps, commonly utilized sealings also cause drag losses based on dry friction. To overcome these challenges, in this contribution, a novel design of sealings for magnetorheological fluid-based actuators is introduced eliminating the adverse drag losses. The intended sealing is based on the magnetorheological fluid itself, positioned in a sealing gap and exposed to a well-defined magnetic stray field of a permanent magnet to prevent any leakage. To eliminate drag torques, a superimposed functional principle with the magnetorheological fluid movement control is utilized. An analytical and a simulation-based modeling approach is proposed describing the torque behavior of the magnetorheological fluid sealing. In experimental investigations, the drag torque-free operation is proven by showing a lossless operation above a defined rotational speed.
\end{abstract}

\section{Keywords}

Magnetorheological fluids, magnetic sealing, magnetorheological fluid sealing, minimization of drag losses, energy-efficient magnetorheological actuator, avoidance of sedimentation

\section{Introduction}

For a further efficiency increase in coupling elements based on magnetorheological fluids (MRFs) applied in many technical sectors, such as in wind turbines (Güth and Mass, 2015) up to modern powertrains of electric and hybrid vehicles (Gratzer et al., 2008; Herber et al., 2016; Smith et al., 2007), a novel sealing design is developed in this contribution. MRFs are smart fluids (Carlson, 2007), consisting of fine magnetic particles in an oil-based carrier fluid, with the particular characteristics of changing their apparent viscosity significantly under the influence of a magnetic field. This property allows the design of mechanical devices for torque transmission, such as brakes and clutches (Maas et al., 2011), with a continuously adjustable and smooth torque generation. Coupling elements based on MRF were already investigated in vehicles as differentials (Gratzer et al., 2008), fan drives for trucks (Smith et al., 2007), or as brakes inside the wheels (Nakano, 2016). Due to the viscous behavior of the MRF, high differential speeds will cause high viscous losses, even in the off-state without an applied magnetic field, as similar occurring in conventional wet clutches (Xiang et al., 2013). Hence, a conventionally MRF-based clutch is not suitable as a starting or shifting clutch in vehicles, where high differential speeds occur.

However, a drag torque-free operation of MRFbased actuators can be achieved by a magnetically induced MRF movement control (Güth and Mass, 2013) resulting in new perspectives regarding the increase in energy efficiency and an improved dynamic response for applications, for example, in powertrains of vehicles, compared to conventional coupling elements. The MRF movement control is based on partially filled shear gaps and on certain volume forces for the movement of the MRF. Thereby, both the avoidance of drag losses and a torque transmission can be realized. In Hegger and Mass (2016b), different concepts of the MRF movement control were investigated. Therein, the conditional stable MRF movement control concept, having no permanent magnets (PMs),

Mechatronic System Laboratory, Technical University of Berlin, Berlin, Germany

\section{Corresponding author:}

Christian Hegger, Mechatronic System Laboratory, Technical University of Berlin, 10623 Berlin, Germany.

Email: christian.hegger@emk.tu-berlin.de 


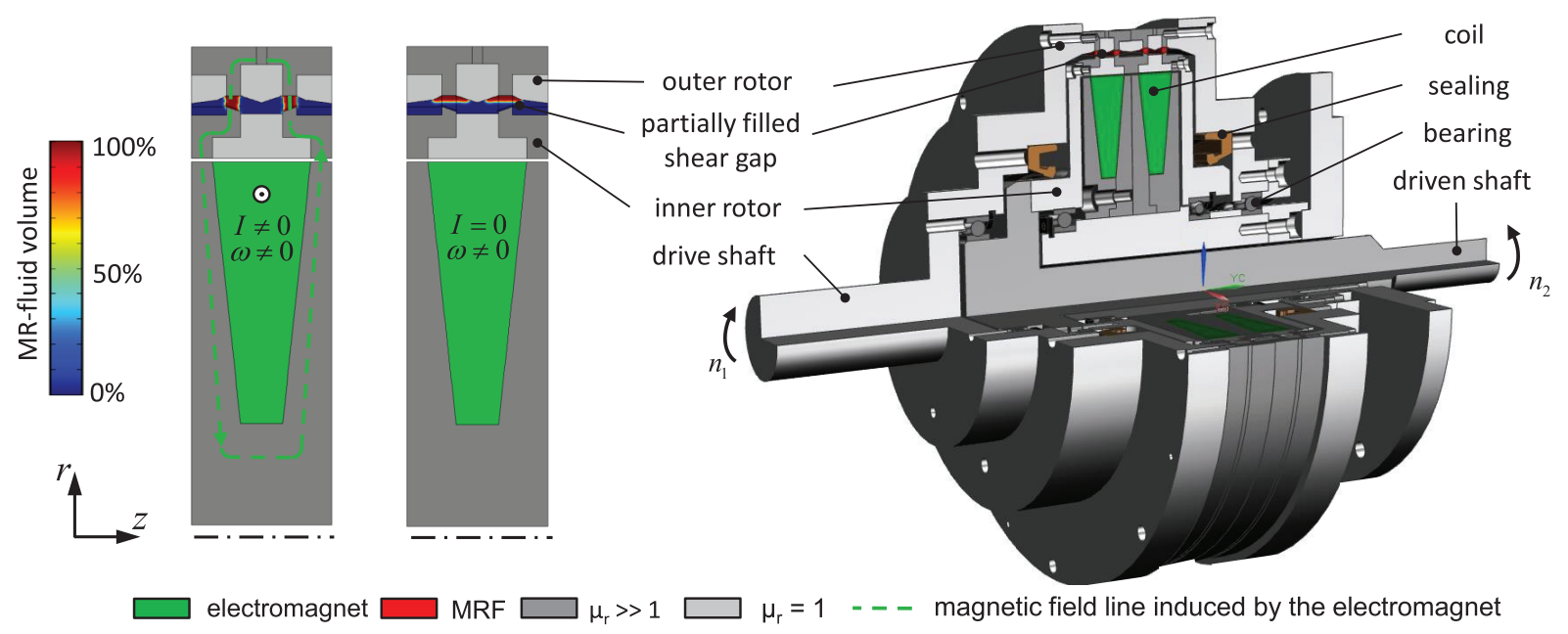

(a)

(b)
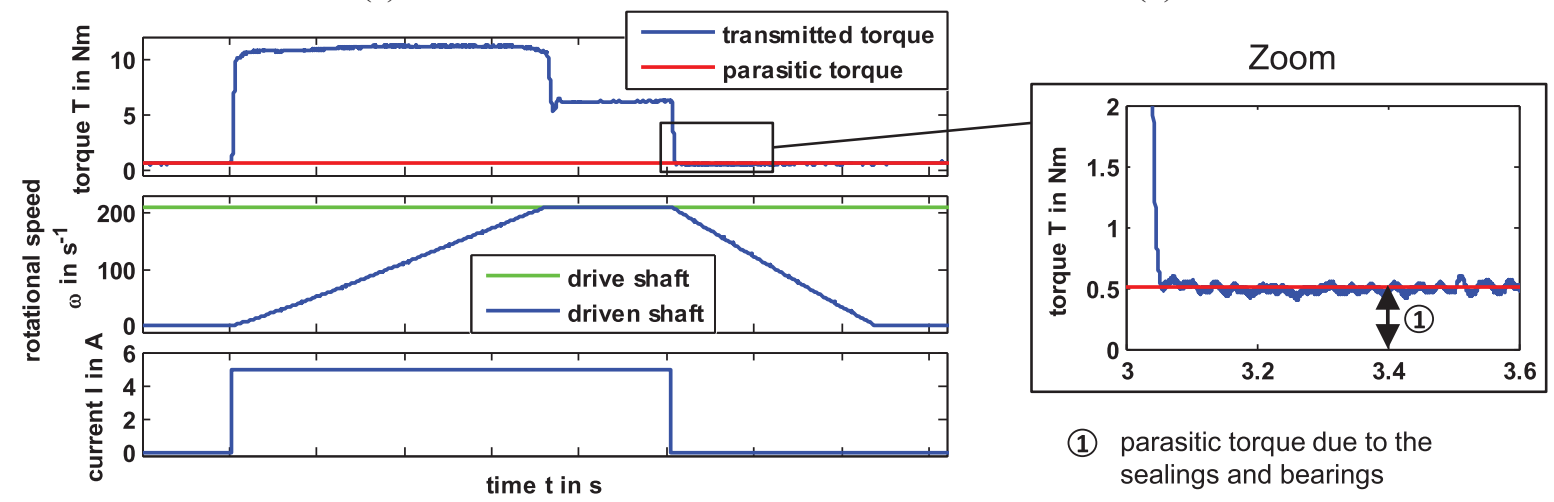

(1) parasitic torque due to the sealings and bearings

(c)

Figure I. Design of the (a) magnetic circuit and (b) mechanical setup of the conditional stable MR fluid movement control concept and (c) measurements of the experimental investigation.

showed the highest capacity of torque which is selected in this contribution. In Figure 1(a), the design of an actuator equipped with the conditional stable MRF movement control is shown. If the electromagnet (EM) is activated by a current of $I \neq 0 \mathrm{~A}$, the MRF will be moved by induced magnetic volume forces to ensure a fluid contact between both rotating surfaces and a torque transmission between the inner rotor and outer rotor. By reducing the current to $I=0 \mathrm{~A}$, the MRF will be moved close to the outer rotor due to rotational acceleration forces to eliminate viscous losses. An experimental investigation of this MRF-based clutch is presented in Figure 1(b). A coupling process is shown engaging the MRF-based clutch to accelerate the driven shaft up to a rotational speed of $n=210 \mathrm{~s}^{-1}$. Disengaging the clutch by reducing the current to $I=0 \mathrm{~A}$ results in a drag torque of $T=0.5 \mathrm{~N} \mathrm{~m}$. The zoom in Figure 1(c) illustrates the avoidance of viscous losses when disengaging the clutch. The remaining drag torque is caused by the parasitic torque of the sealings and bearings, as shown in Figure 1(b). Reducing this drawback and thus increasing the energy efficiency even further will be the challenging task of this contribution. Applying magnetic fluid-based sealings instead of conventional nitrile butadiene rubber (NBR)-based radial sealings is already state of the art and applied in several areas. Sealings based on ferro fluids (FFs; Borbath et al., 2010) are commonly used for systems requiring a high vacuum integrity and hermeticity. Lifetime, dynamic range and parasitic drag torque are considerable advantages compared to conventional sealings. They are applied in hard disk drives (HDD), $\mathrm{CO}_{2}$ lasers, high-power X-ray tubes, and many more applications (Eagle Industry Co., Ltd, 2014; Raj and Moskowitz, 1990). The design of a magnetic sealing is based on an axial sealing gap in which the corresponding magnetic fluid is exposed to the field of a PM. Further design and material constrains are explained in detail in Borbath et al. (2011). Nevertheless, the combination of FFs for sealing and MRFs for torque transmission in the same shear gap seems not to be applicable. Furthermore, sealings based on MRFs are 
also under investigation. In Kordonski and Gorodkin (1996), a rotational sealing based on magnetic fluids was realized. Therein, the behavior of FF and MRF was investigated separately. The experimental investigation shows many characteristic differences of the two applied magnetic fluids. The most decisive difference is the higher achievable critical pressure and the significant higher drag torque at certain rotational speeds of the MRF sealing. In Potoczny and Zachara (2012), the influence of the amount of MRF volume of a radial MRF sealing on the critical pressure was investigated under the influence of certain rotational speeds and sealing gap heights. Thereby, critical pressures up to 1.8 bar were measured. The mentioned contributions dealt with magnetic fluid sealings to separate gas volumes. In Lampe and Thess (1998) and Lampe (2000), a MRF sealing for a MRF-based clutch was experimentally validated showing a good accordance with an introduced model. The design of the sealing is based on a tapered gap. The tightness under the impact of longer application downtimes and the torque behavior under the influence of rotational speeds were not investigated. In all contributions mentioned before, the magnetic excitation system consists of PMs.

In this contribution, a sealing based on MRFs will be designed and investigated. The novel approach will eliminate the drag losses ascribed to conventional sealings, on one hand, and the viscous-induced torque of the MRF in combination with the MRF movement control, on the other hand. After introducing the design and functional principle of the MRF sealing, an analytical modeling approach for the calculation of the required rotational speed ensuring a drag torque-free operation of the sealing is presented. The experimental investigation shows the feasibility and restrictions of the MRF sealing design. Based on this insights, an extended design of the MRF sealing is proposed by finite element analysis (FEA) of the torque behavior. To prove the elimination of viscous- and frictionalinduced drag torques, on one hand, and the tightness of the smart sealing during longer downtimes, on the other hand, experimental investigations based on an optimized sealing design are finally performed.

\section{Fundamental MRF sealing}

In this section, the functional principle and a design of the smart MRF sealing are introduced. In addition, an experimental investigation using a simple test setup is presented.

\section{Design}

In Figure 2, the schematic design of a MRF sealing for A MRF-based clutch equipped with a conditional stable MRF movement control is shown. In Figure 2(a),

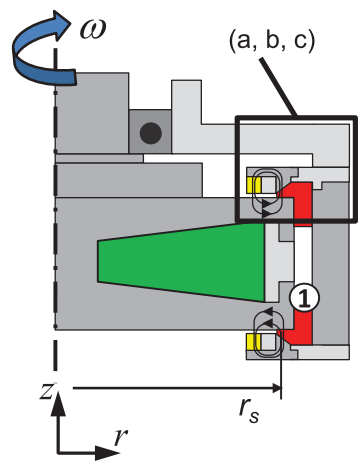

(1) partially filled shear gap

(2) radial sealing gap

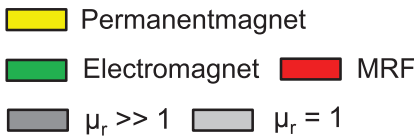

(a)

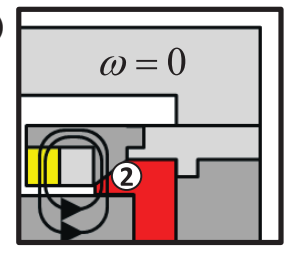

(b)

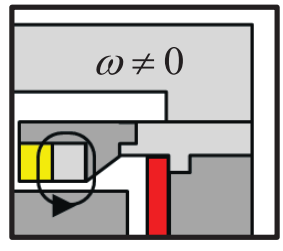

(c)

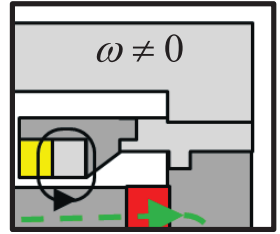

Figure 2. Schematic design of the smart MRF sealing and distribution of the MRF (a) during standstill, (b) at rotational speed $\omega_{c}$, and (c) during torque transmission.

the clutch is in standstill, and the MRF moves toward the smart sealing due to the impact of gravity. Due to the magnetic field of the PM, the yield stress of the MRF is increased in the area of the radial shear gap. Therefore, leakage is avoided. Under the impact of higher rotational speeds (Figure 2(b)), the MRF will be moved out of the sealing gap and placed at the outer rotor by radial acceleration forces. Similar to the conditional MRF movement control, drag torque can be completely eliminated. Thus, the mentioned drawback in Kordonski and Gorodkin (1996) of the MRF sealing describing viscous-increased shear stress at higher shear rates is avoided. Activating the EM in Figure 2(c) results in a movement of the MRF from the outer rotor toward the inner rotor to engage the clutch according to the MRF movement control in Güth et al. (2013). Consequently, the MRF is utilized to transmit torque, on one hand, and to realize a sealing, on the other hand, ensuring an energy-efficient operation.

To realize the drag torque-free operation of the sealing, a critical speed $\omega_{c}$ has to be achieved. This critical speed $\omega_{c}$ depends on the design of MRF-based actuators and can be calculated by considering the following force equilibrium

$$
\mathbf{f}_{\tau}-\mathbf{f}_{a}=0
$$

Therein, $\mathbf{f}_{\tau}$ represents the volume force caused by the yield stress and $\mathbf{f}_{a}$ is the volume force induced by radial acceleration. The volume force $\mathbf{f}_{\tau}$ can be determined by the stress tensor $\mathbf{S}$, describing the shear stress in an infinitesimal element in the sealing gap, as shown in Figure 3 


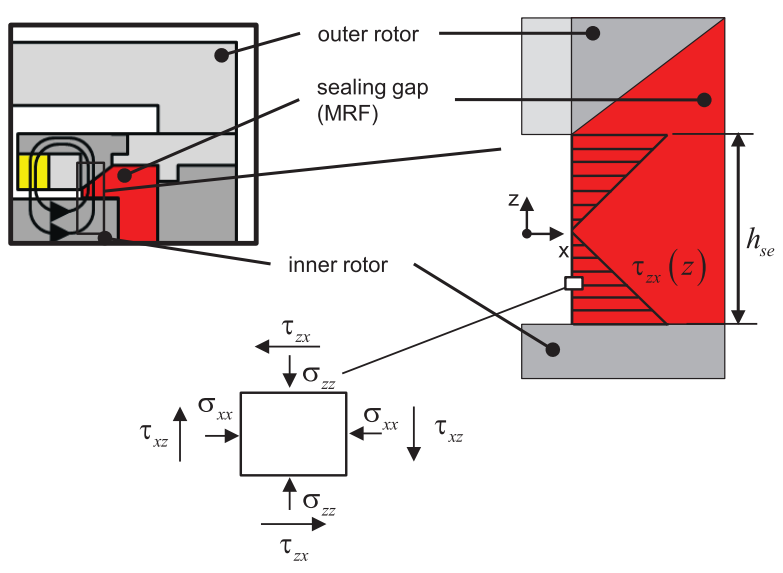

Figure 3. Sectional view of the schematic design of the sealing gap showing the distribution of the effective shear stress $\tau_{z x}$.

$$
\mathbf{S}=\left(\begin{array}{cc}
\sigma_{x x} & \tau_{x z} \\
\tau_{z x} & \sigma_{z z}
\end{array}\right)
$$

Therein, $\sigma$ represents the stress and $\tau$ is the shear stress according to the Cartesian coordinates $x$ and $z$. The divergence of the stress tensor $\mathbf{S}$ results in a volume force which is based on the magnetically induced stress and shear stress

$$
\nabla \mathbf{S}=\left(\begin{array}{c}
\frac{\partial}{\partial x} \sigma_{x x}+\frac{\partial}{\partial z} \tau_{z x} \\
\frac{\partial}{\partial x} \tau_{x z}+\frac{\partial}{\partial z} \sigma_{z z}
\end{array}\right)=\mathbf{f}_{\tau}
$$

In the sealing gap, the stress $\sigma_{x x}, \sigma_{z z}$, and the shear stress $\tau_{x z}$ can be assumed to be close to zero. The volume force due to radial acceleration can be determined as

$$
\mathbf{f}_{a}=\left(\begin{array}{l}
\omega_{c}^{2} \cdot r_{s} \cdot \rho \\
0
\end{array}\right)
$$

The parameter $r_{s}$ is the radius of the sealing gap and $\rho$ is the density of the MRF $\left(4.2 \mathrm{~g} / \mathrm{cm}^{3}\right)$. Considering the remaining shear stress $\tau_{z x}$ in equation (3) and the radial acceleration from equation (4) results in

$$
\frac{\partial}{\partial z} \tau_{z x}=\omega^{2} \cdot r_{s} \cdot \rho
$$

According to Figure 3, the effective shear stress $\tau_{z x}$ in this approach depends on the $z$-coordinate. Under consideration of the following expression, the shear stress $\tau_{z x}$ at the smallest height $h_{s e}$ of the sealing gap can be calculated by

$$
\tau_{z x}(z)=\frac{2|z|}{h_{s e}} \cdot \tau_{0}\left(B_{z}\right), \quad-\frac{h_{s e}}{2} \leq z \leq \frac{h_{s e}}{2}
$$

According to Figure 4, the magnetic flux density in $z$-direction can be assumed as almost constant, yielding $\partial B_{z} / \partial z \approx 0$. Solving equation (5) with $\omega=\omega_{c}$ under consideration of equation (6), the critical speed results in

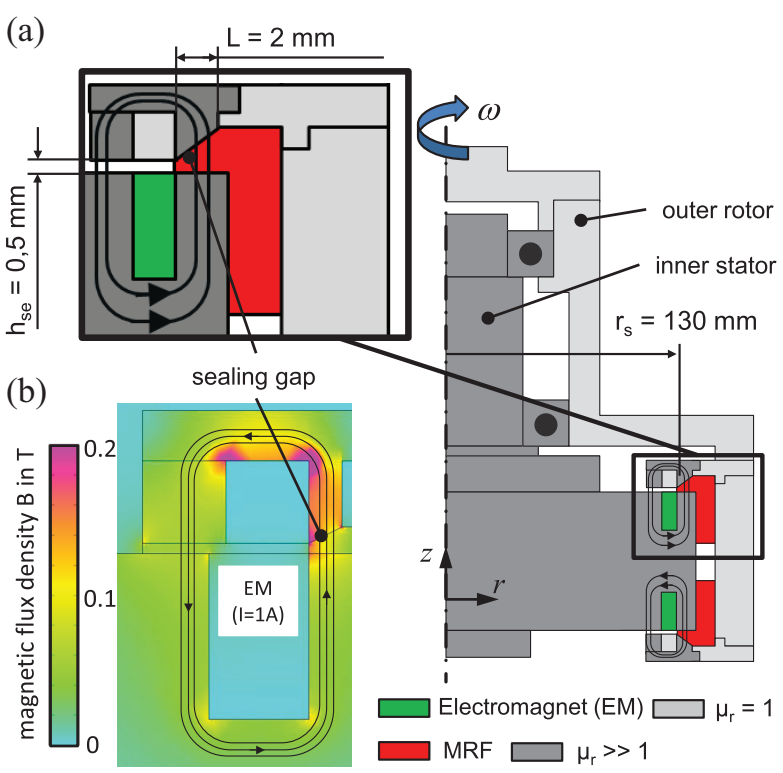

Figure 4. (a) Design of the test actuator with enlarged sections of the schematic design and (b) the distribution of the magnetic flux density $B$ of the MRF sealing.

$$
\omega_{c}=\sqrt{\frac{2}{h_{s e} \cdot r_{s} \cdot \rho} \cdot \tau_{0}\left(B_{z}\right)}
$$

Consequently, the critical speed $\omega_{c}$ depends on the height $h_{s e}$ and effective radius $r_{s}$ of the sealing, the applied type of MRF, and the magnetically induced yield stress $\tau_{0}$. This approach neglects the influence of fluid dynamics and shear rates.

\section{Experimental investigation}

The first experimental investigation was performed with a simple MRF-based test actuator illustrated in Figure 4. The design is based on an inner stator and an outer rotor. In contrast to the design in Figure 2, the central positioned EM for the torque transmission and the PMs for the sealings are removed. Instead, each sealing is realized by an EM which enables an investigation of the MRF sealing in a wide range of operation. The radius of the sealing gap is set to $r_{s}=130 \mathrm{~mm}$ representing the radius of a full-scale MRF-based clutch in hybrid powertrains. In Figure 4(b), the distribution of the magnetic flux density $B$ is shown. The flux density in the sealing gap is diminished under the impact of the increasing sealing gap height $h_{s e}$. The dynamic pressure of the sealing is approximately calculated by (Coulter et al., 1993; Zhao et al., 2016)

$$
\Delta p=\int_{0}^{L} \frac{c \cdot \tau\left(B_{z}(r)\right)}{h_{s e}(r)} d r
$$


The parameter $c=3$ considers the viscosity class of the MRF. The length of the sealing gap is defined by the parameter $L$. The calculation of the dynamic pressure results in $\Delta p_{c}=0.183$ bar at a current of $I=1 \mathrm{~A}$, which corresponds very well with the measured dynamic pressure during standstill of $\Delta p_{m}=0.19$ bar. Assuming a degree of filling of $\beta=50 \%$, the maximum occurring pressure during standstill at the sealing is about $\Delta p_{\max }=0.06$ bar. Thus, the achievable dynamic pressure of the MRF sealing is sufficient. Next, the characteristics of the drag torque will be investigated.

In Figure 5, the results of the drag torque measurements are illustrated. During the measurement, the rotational speed is slowly increased from $\omega=0 \mathrm{~s}^{-1}$ to $\omega=415 \mathrm{~s}^{-1}$ ensuring a steady-state operation. Within the acceleration up to a rotational speed of $\omega=30 \mathrm{~s}^{-1}$, the drag torque increases due to the shear stress enhanced by the increasing shear rate. Under the impact of further increasing speeds, the measured torque $T$ drops down to the parasitic torque of the bearings. Thereby, higher currents $I$ of the EM require a higher rotational speed $\omega_{c}$. Consequently, the MRF is moved out of the sealing gap and is superimposed with the behavior of the conditional stable MRF movement control. The value of the marked analytically calculated critical rotational speed $\omega_{c}$ (equation (7)) for the corresponding currents $I$ occurs always beyond the initial drop of the measured torque $T$.

This can be reduced to the MRF in the sealing gap at higher shear gap heights $h_{s e}$ where the MRF is

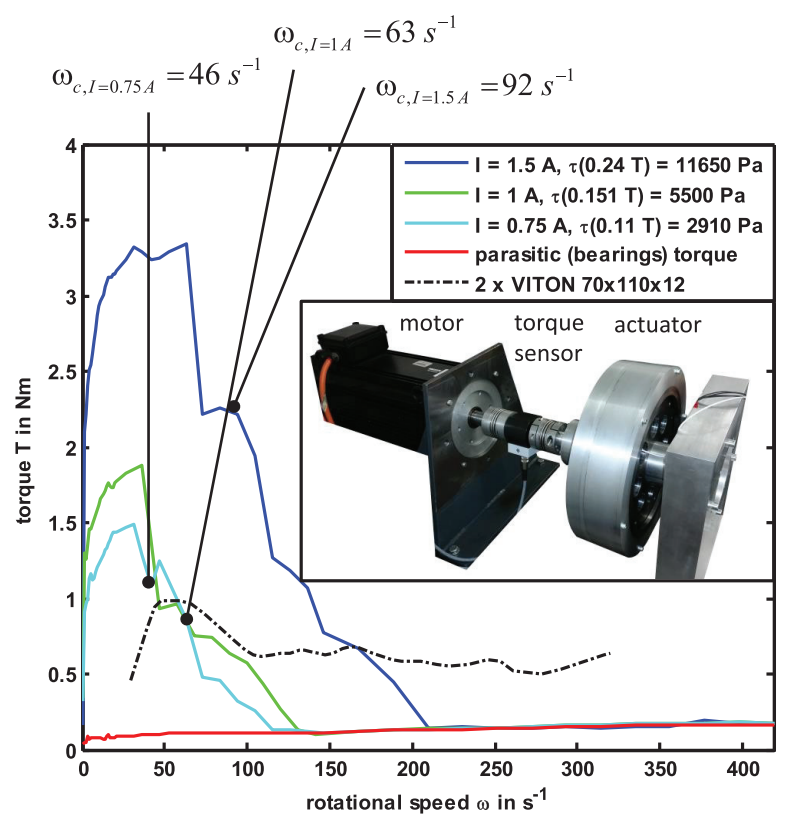

Figure 5. Steady-state measurement results of the drag torque of the MRF test actuator equipped with two MR fluid sealings under the impact of certain currents $I$ and a speed ramp of $2666 \mathrm{r} / \mathrm{min}$. exposed to a lower magnetic flux density. This volume will be moved out of the sealing gap at first. However, the measured rotational speed for a drag torque-free operation is approximately two times higher compared to the calculated critical rotational speed $\omega_{c}$. This can be explained due to the neglected fluid dynamics and a changing magnetic behavior. Due to the already removed volume of the MRF during the initial drop, the magnetic reluctance of the sealing gap has been changed, and the magnetic flux density, respectively, yield stress $\tau_{0}$, in the remaining MRF is enhanced. Additionally, the shear rate also increases the shear stress of the MRF in the sealing gap. Although simplifications and neglections are made to derive equation (7), the analytically calculated critical speed $\omega_{c}$ indicates the range of the rotational speed for a drag torque-free operation. For a more detailed calculation, simulations based on FEA will be introduced later. Figure 5 contains also the drag torque caused by two standard radial shaft sealings (Silvestri et al., 2006) in dependency on the rotational speed. A similar increase and drop of the drag torque under the impact on increasing rotational speeds is observed, except that the torque remains at $T \approx 0.6 \mathrm{~N} \mathrm{~m}$ at $\omega \geq 1000 \mathrm{~s}^{-1}$. It has to be considered that the radius of the radial shaft sealing is only $r_{s}=35 \mathrm{~mm}$, which explains the smaller torque at the beginning of the measurement compared to the MRF sealing with $r_{s}=130 \mathrm{~mm}$. A radius similar to the MRF sealing would result in an enormous drag torque of more than $7 \mathrm{~N} \mathrm{~m}$, and in addition, the maximum sliding speed will be exceeded. Instead, the MRF sealing holds the MRF directly in the shear gap by which the response time can be increased during system starts.

\section{Tightness}

During the experimental investigation, leakage did not occur, and the MRF sealings were tight. However, after 2 weeks of standstill, a leakage of the carrier fluid of the MRF at the lower area of the actuator was observed, see Figure 6(c). This can be ascribed to the sedimentation of the MRF. Under the impact of gravity, the iron particles sediment in the shear gap, while the carrier liquid accumulates in the upper area of the shear gap. The pure carrier liquid passes the sealing and gathers at the lowest area outside of the sealing, as illustrated in Figure 6(b). A closer look at the structure of the MRF sealing reveals the reason for the leakage. The dynamic pressure of a MRF sealing consists of two components, as investigated in Fujita et al. (1999). On one hand, the dynamic pressure is based on the magnetically induced yield stress of the particle chains. On the other hand, the carrier liquid is located and hold by capillary forces in the spaces between the particle chains which contribute to the dynamic pressure as well. The pressure applied on a homogeneous particle 


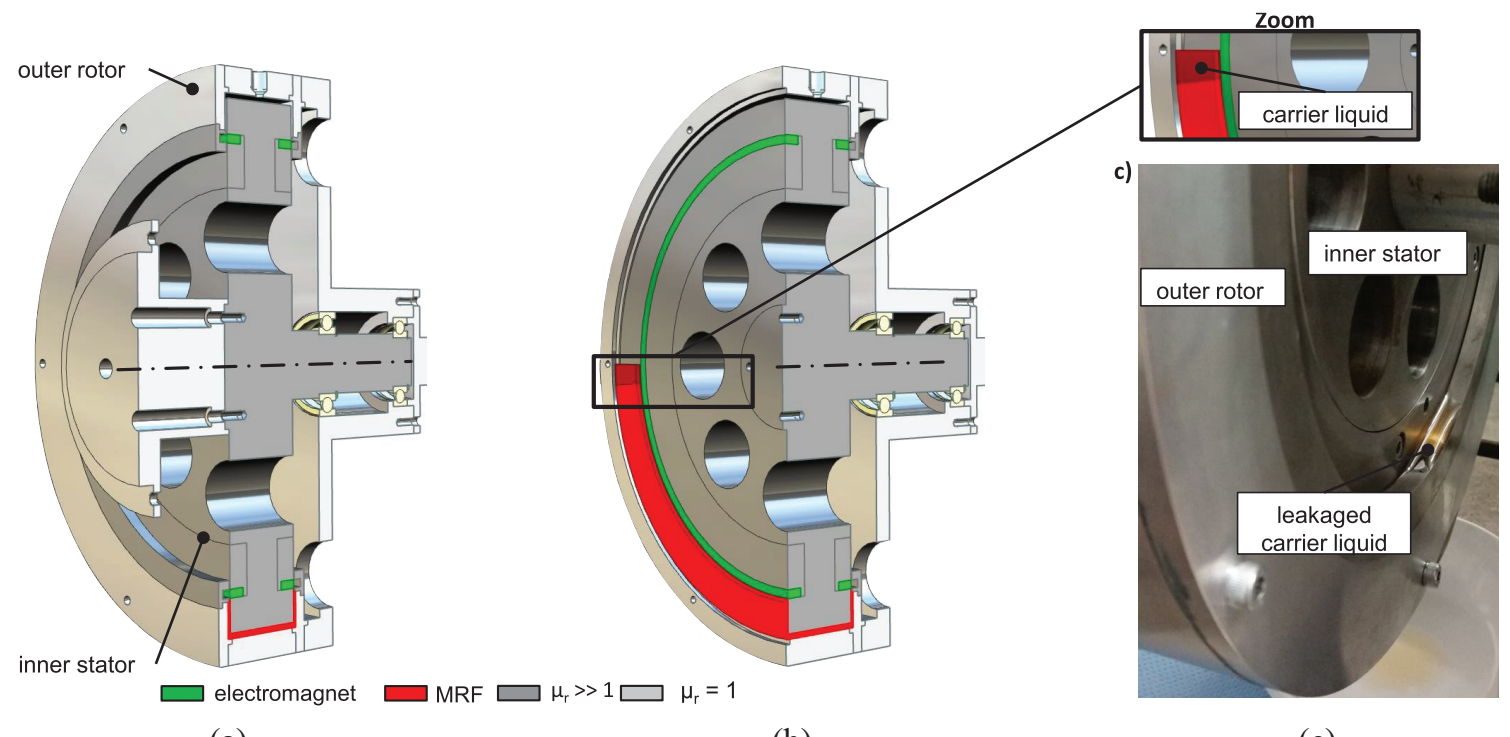

(a)

(b)

(c)

Figure 6. (a) Investigation of the tightness based on the half section of the MRF test actuator, showing the (b) distribution and sedimentation of the MRF during longer standstill and (c) a picture of the leakage of the carrier liquid.

distribution of the MRF affects mainly the chain structure of the MRF in the sealing gap. Instead, the pure carrier liquid affects equally on both components. Due to the capillary forces, the much weaker resistance forces cannot prevent the leakage of the pure carrier liquid through the spaces between the particle chains. To increase the effect of the capillary force, the length $L$ of the sealing gap can be extended. But increasing the length $L$ would result in a higher drag torque which may not be compensated during the acceleration phase. Since there is no application without downtimes, the tightness may be improved by changing the viscosity of the carrier liquid or by reducing the size of the iron particles. But changing the characteristics of the MRF is also not applicable. Thus, a new design of a MRF sealing is introduced in the next section.

\section{Optimized MRF sealing}

During the previous investigation, a leakage of the carrier fluid was observed after longer downtimes. In this section, an optimized MRF sealing will be presented to ensure the tightness at any downtime.

\section{Design}

The investigated leakage of the carrier fluid was caused by the sedimentation of the iron particles in the MRF within the shear gap. Thus, in this approach, the sedimentation of the MRF is intended to be prevented by a magnetically increased yield stress of the MRF. In Vicente et al. (2000), the effect of a magnetic field on the sedimentation behavior of a suspension similar to MRFs is already investigated. Therein, probes were exposed to a slightly vertical magnetic field $(B=2.5 \mathrm{mT})$. The impact of the applied magnetic field cannot prevent the sedimentation, but the speed of sedimentation was decreased significantly. In this approach, the magnetic field will be increased to create stable MRF chain structures in the shear gap, in order to prevent the sedimentation completely. For the calculation of the required magnetic field, two modeling approaches will be presented. It is assumed that the capillary force of the carrier liquid between the particle chains is sufficient to resist the impact of the own weight during standstill. The first approach is based on the dipole-dipole model (Güth and Mass, 2016) by which the interaction force between the particles in the chain structure can be calculated by

$$
\begin{aligned}
\mathbf{F}_{j}^{i}= & \frac{8}{3} \frac{\mu_{0} \cdot \pi \cdot r_{p}^{6} \cdot \chi_{e f f}^{2}}{r_{i j}^{5}} \cdot H_{0}^{2} \cdot \sum_{j \neq i}^{n} \\
& \left\{\left[\cos \theta\left(r_{i j}+\frac{2}{3 r_{i j}^{2}} \chi_{e f f} \cdot r_{p}^{3}\right)\right]\right. \\
& \left.\hat{\mathbf{k}}-\left[\cos ^{2} \theta \cdot\left(3+\frac{2}{r_{i j}^{3}} \chi_{e f f} \cdot r_{p}^{3}\right)\right] r_{i j} \cdot \hat{\mathbf{e}}_{r}\right\}
\end{aligned}
$$

The parameter $\mu_{0}$ describes the permeability of free space, $r_{p}$ is the radius of the particle, $r$ is the distance between two particles $j$ and $i, H_{0}$ is the applied magnetic field in the shear gap, $\hat{k}$ is the position vector of the magnetic field, $\hat{e}$ is the position vector of the distance between the particles, and $n$ is the number of particles in the chain. The magnetic susceptibility $\chi_{\text {eff }}$ is 
described by the Froehlich-Kennelly relation to consider the nonlinear magnetic behavior of the particles

$$
\chi(H)=\frac{\chi_{0} \cdot M_{s}}{\chi_{0} \cdot M_{s}+H}
$$

Therein, $\chi_{0}$ is the initial susceptibility with $\chi_{0}=1000$, $M_{s}$ is the saturation magnetization with $M_{s}=2.1 \mathrm{~T} /$ $\mu_{0}$, and $H$ is the magnetic field inside the particle. The approach is applied for the first and second areas in the shear gap, compare Figure 7. In the first area, the particle chains are not sheared $(\theta=0)$ during standstill, and the direction of the magnetic interaction force $f_{m}$, Pos 1 of the homogeneous distributed particle chains is longitudinal to the direction of the magnetic field, see Figure 7(a). Consequently, equation (9) can be simplified to

$$
\begin{aligned}
& f_{m, P o s}=\frac{8}{3} \frac{\mu_{0} \cdot \pi \cdot r_{p}^{6} \cdot \chi_{e f f}^{2}}{r_{i j}^{5}} \cdot H_{0}^{2} \cdot \sum_{j \neq i}^{n} \\
& \left\{\left[\left(r_{i j}+\frac{2}{3 r_{i j}^{2}} \chi_{e f f} \cdot r_{p}^{3}\right)-\left[\left(3+\frac{2}{r_{i j}^{3}} \chi_{e f f} \cdot r_{p}^{3}\right)\right] r_{i j}\right]\right\}
\end{aligned}
$$

In the second area of the shear gap, the direction of the gravity is orthogonal to the direction of the magnetic field, see Figure 7(b). Under consideration of the orthogonal direction, the magnetically induced reaction force $f_{m}$, Pos 2 can be calculated by equation (9), according to Güth and Mass (2016)

$$
\begin{gathered}
f_{m, \text { Pos } 2}=\frac{8}{3} \cdot \mu_{0} \cdot \pi \cdot r_{p}^{6} \cdot \chi_{\text {eff }}^{2} \cdot H_{0}^{2} \cdot \cos ^{2}(\theta) \cdot \sin (\theta) . \\
\sum_{j \neq i}^{n} \frac{1}{r_{i j}^{4}}\left(3+\frac{2}{r_{i j}^{3}} \chi_{\text {eff }} r_{p}^{3}\right)
\end{gathered}
$$

Therein, $\theta$ describes the angle between the magnetic field and the chain and is defined by the relation of $\gamma=\tan (\theta)$. The shear strain $\gamma$ is determined by 0.3 , close to the maximum value. To determine the relationship between the applied magnetic flux density $B$ in the shear gap, the averaged applied magnetic field $H_{0}$ and the magnetic field inside the particle $H$, a numerical calculation of the magnetic field has been carried out for an homogeneous particle volume concentration of $c_{V}=0.47$ as shown in Figure 8. In addition, Figure 8 shows also the longitudinally magnetic-induced interaction force $f_{m}$, Pos 1 in area 1 , the orthogonally magneticinduced interaction force $f_{m}$, Pos 2 in area 2 of a particle chain, and the reaction force due to the gravity $f_{g}$. A magnetic flux density of $B>5 \mathrm{mT}$ is already sufficient to achieve a stable chain structure in the first area in the shear gap avoiding the sedimentation. In the second

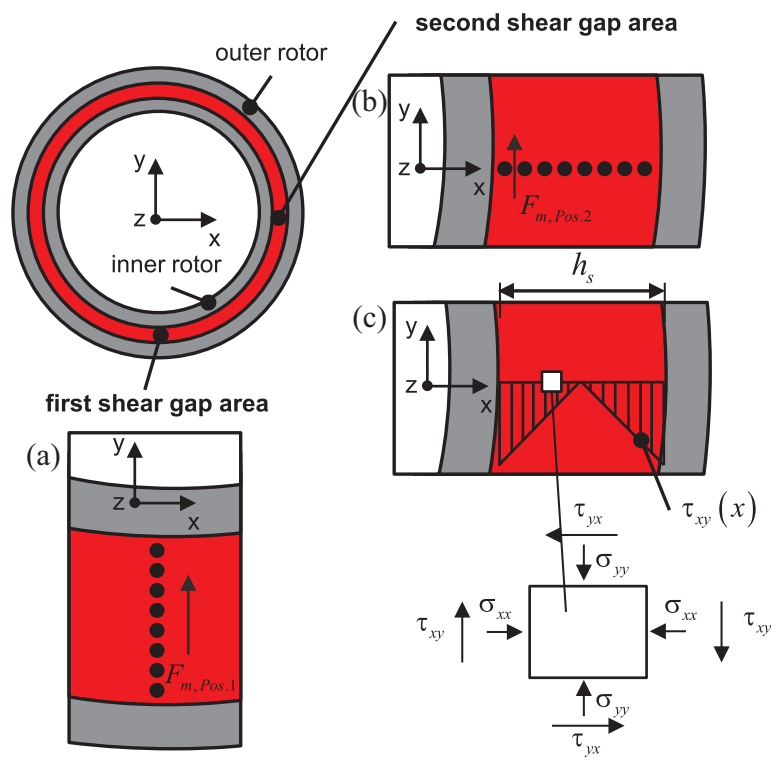

Figure 7. Schematic design of the shear gap showing (a) the longitudinally magnetic-induced interaction force $f_{m}$, Posl, (b) the orthogonal magnetically induced interaction force $f_{m}$, Pos 2 , and (c) the distribution of effective shear stress $\tau_{x y}$.

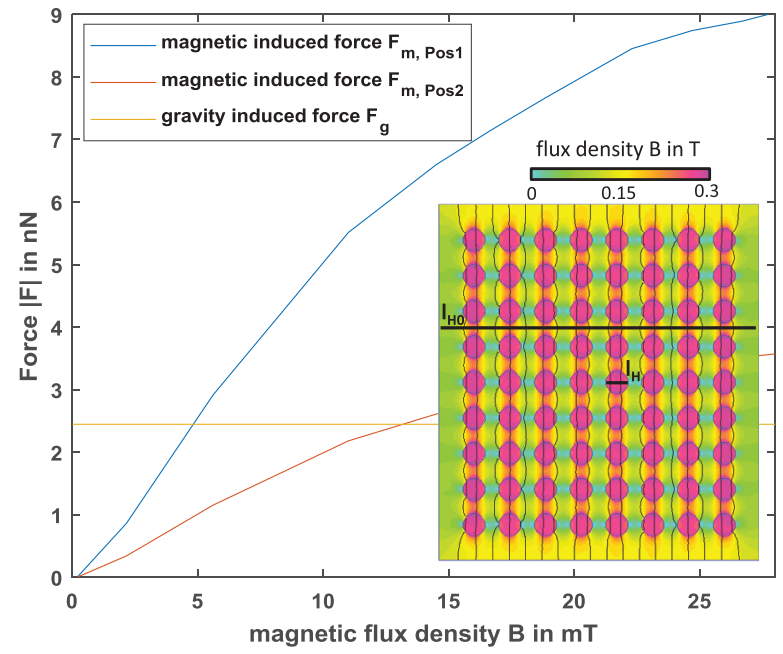

Figure 8. Calculated interaction forces between the particles and the distribution of the magnetic flux density of the chain structure.

area of the shear gap, at least a magnetic flux density of $B>14 \mathrm{mT}$ is required.

In addition, the second approach based on the yield stress $\tau_{0}(B)$ of the continuum given by equations (1) and (3) is presented to prove the obtained results of the previous approach. Since in this case no movement and a homogeneous distribution of the MRF can be expected, this modeling approach is also applicable. The equation for the volume force due to radial accelerations $\mathbf{f}_{a}$ is, therefore, replaced by the volume force $\mathbf{f}_{g}$, given by 


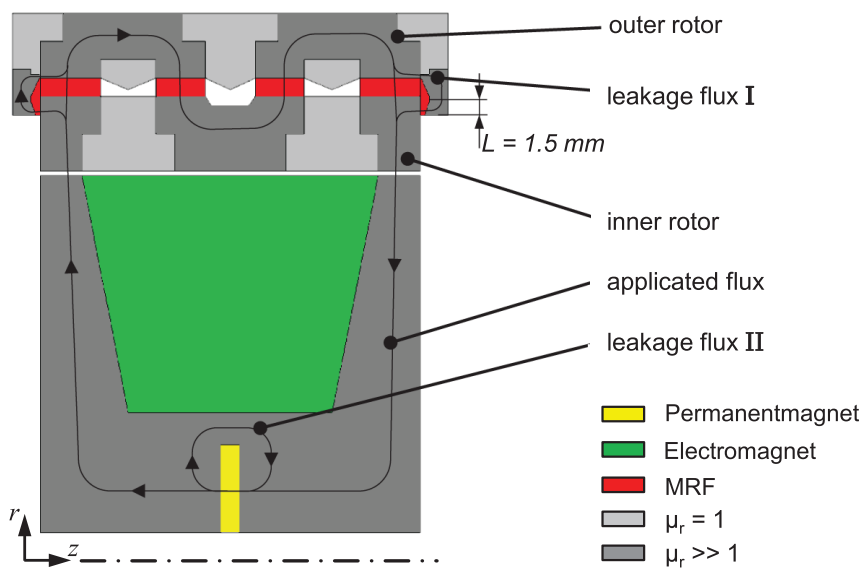

(a)

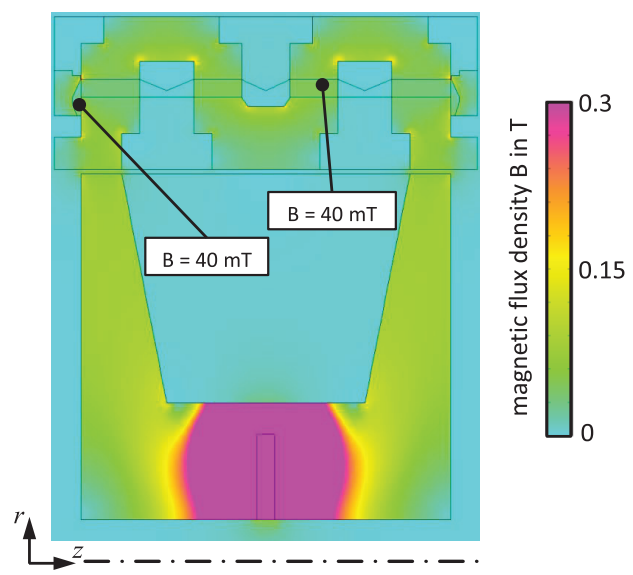

(b)

Figure 9. Schematic (a) design and (b) distribution of the magnetic flux density of the improved MRF test actuator for an optimized MR fluid sealing effect.

$$
\mathbf{f}_{g}=\left(\begin{array}{l}
0 \\
g \rho
\end{array}\right)
$$

to consider the influence of the gravity. To ensure a sufficient value of shear stress, this approach considers the horizontal plane in the second area (Figure 7(c)) of the shear gap where due to gravity effects, the highest load on the shear stress occurs

$$
\tau_{x y}(x)=\frac{2|x|}{h_{s}} \cdot \tau_{0}\left(B_{x}\right), \quad-\frac{h_{s}}{2} \leq x \leq \frac{h_{s}}{2}
$$

The horizontal plane and the according parameters are declared in Figure 7. The flux density distribution in the $x$-direction can be assumed as almost constant, $\rightarrow \frac{\partial B_{x}}{\partial x} \approx 0$, which results in

$$
\tau_{0}\left(B_{x}\right)=g \rho \cdot \frac{h_{s}}{2}
$$

Therein, $g$ is gravity and $h_{s}$ is the shear gap height (2 mm). According to equation (15), a shear stress of $\tau=42 \mathrm{~Pa}$ is required to withstand the impact of gravity which corresponds to a magnetic flux density of $B=18 \mathrm{mT}$ confirming the obtained results of the previous approach. The modeling approaches show, on one hand, that in the first region, already a magnetic flux density of $B=5 \mathrm{mT}$ ensures sufficient interaction forces between the particles to withstand the impact of gravity and, consequently, the sedimentation. In the second area where the highest physical stress occurs, a magnetic field strength of at least $B=14 \mathrm{mT}$ is required.

The magnetic flux density in the final shear gap design will be chosen to be $B=40 \mathrm{mT}$ to ensure a sufficient safety factor. In Figure 9(a), the MRF test actuator with an optimized design of the MRF sealing is illustrated. The number of PMs is reduced to one. The remaining $\mathrm{PM}$ is positioned within the inner magnetic circuit below the center of the EM and ensures a sufficient magnetic flux density in the shear gap to avoid sedimentation. The design of the integrated PM causes the leakage flux II. By an FEA, it was proven that this design requires the lowest feeding power for the torque transmission mode. Besides, this design allows a simple mechanical construction of the stationary excitation system. The magnetic flux in the sealing gap to ensure the tightness is based on the enforced leakage flux $\mathbf{I}$. Since, the MRF in the shear gap is "freezed" by the PM, the high dynamic pressure almost vanishes in the sealing gap when compared to the previous design in Figure 4. Consequently, the magnetically induced yield stress, respectively, magnetic flux density, in the sealing gap can be lowered to $B=40 \mathrm{mT}$, see Figure 9(b). Despite the main volume of the MRF in the shear gap is "freezed" by the PM, some MRF in the region between the sealing and shearing gap is not sufficiently exposed to the magnetic flux due to the construction. Thus, these MRF volume parts will still move into the sealing gap during standstill and cause drag torques in the acceleration phase. The design of the proposed MRF test actuator in Figure 9 is based on a serpentine flux guidance reducing weight, space, and excitation energy (Hegger and Mass, 2016a). In addition, the design of the sealing gap was adjusted to reduce the drag torque during the acceleration phase. Especially, the sealing gap length was reduced to $L=1.5 \mathrm{~mm}$. Furthermore, a two-dimensional FEA simulation was performed to determine the drag torque behavior of the sealings. Therein, the movement of the MRF is calculated by Navier-Stoke's equation with the additional term $\mathbf{f}_{\tau}$ to consider the volume force due to the magnetically increased shear stress 

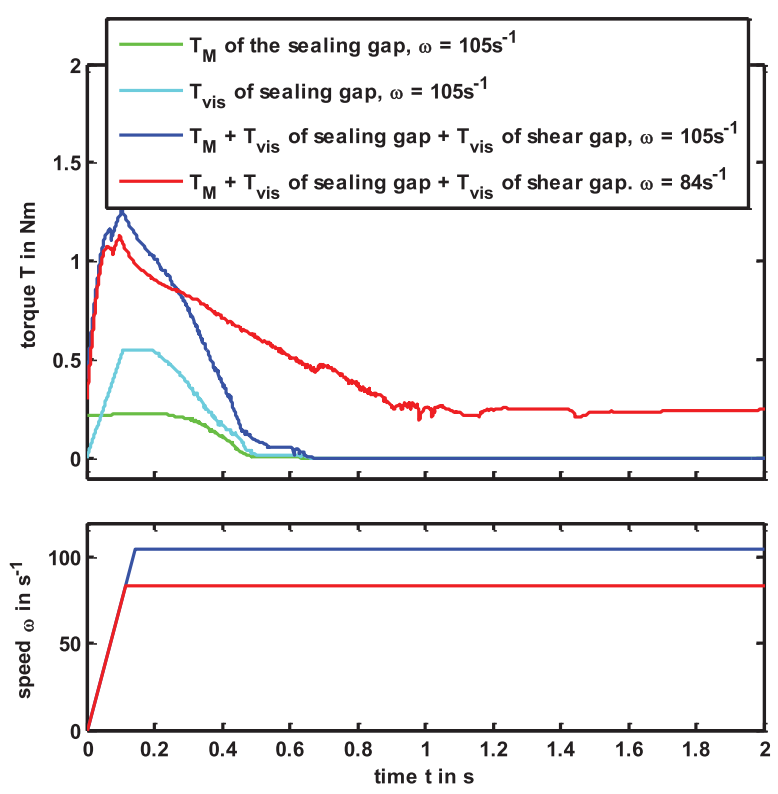

Figure 10. Simulation of the torque behavior of the improved MRF test actuator for different rotational speeds $\omega$.

$$
\rho \cdot\left(\frac{\partial v}{\partial t}+(\mathbf{v} \cdot \nabla) \mathbf{v}\right)=\eta \cdot \Delta \mathbf{v}+\mathbf{f}_{g}+\mathbf{f}_{a}+\mathbf{f}_{\tau}
$$

Therein $\rho$ describes the density of the MRF, $v$ is the velocity field, $g$ is the acceleration due to gravity, $\omega$ is the angular velocity, and $r$ is the position of the MRF. Since in the transient simulation, the movement of the MRF is utilized, the shear rate $\dot{\gamma}$ has to be considered for the calculation of the shear stress $\tau$

$$
\tau(\dot{\gamma}, B)=\tau_{M}+\tau_{v i s}=\tau(|B|)+\eta \cdot \dot{\gamma}=\tau(|B|)+\eta \cdot \nabla \mathbf{v}
$$

The shear stress of the MRF $\tau(\dot{\gamma}, B)$ is based on the magnetically induced yield stress $\tau_{M}$ and the viscousinduced shear stress $\tau_{v i s}$. To determine this quantities in a two-dimensional rotationally symmetric simulation, equation (3) is transformed into cylindrical coordinates and extended by the shear rate

$$
\mathbf{f}_{\tau}=\left(\begin{array}{c}
\left(\frac{\partial \sigma_{r r}}{\partial r}+\frac{\sigma_{r r}}{r}\right) \cdot \operatorname{sgn}\left(\frac{\partial v_{r}}{\partial r}\right)+\frac{\partial \tau_{z r}}{\partial z} \cdot \operatorname{sgn}\left(\frac{\partial v_{r}}{\partial z}\right) \\
\left(\frac{\partial \tau_{r z}}{\partial r}+\frac{\tau_{r z}}{r}\right) \cdot \operatorname{sgn}\left(\frac{\partial v_{z}}{\partial r}\right)+\frac{\partial \sigma_{z z}}{\partial z} \cdot \operatorname{sgn}\left(\frac{\partial v_{z}}{\partial z}\right)
\end{array}\right)
$$

Hence, the simulation is based on fluid dynamics; the correct sign of the volume force $\mathbf{f}_{\tau}$ and direction of the fluid movement are considered according to the shear rate parts $\partial v / \partial r$ and $\partial v / \partial z$ and the sgn function. The volume force $\mathbf{f}_{\tau}$ is implemented in the simulation software COMSOL Multiphysics 4.4 to investigate the drag torque behavior of the sealing gap for different rotational speeds $\omega$. In Figure 10, the calculated torque behavior of the optimized design is presented for certain rotational speeds. The calculation of the

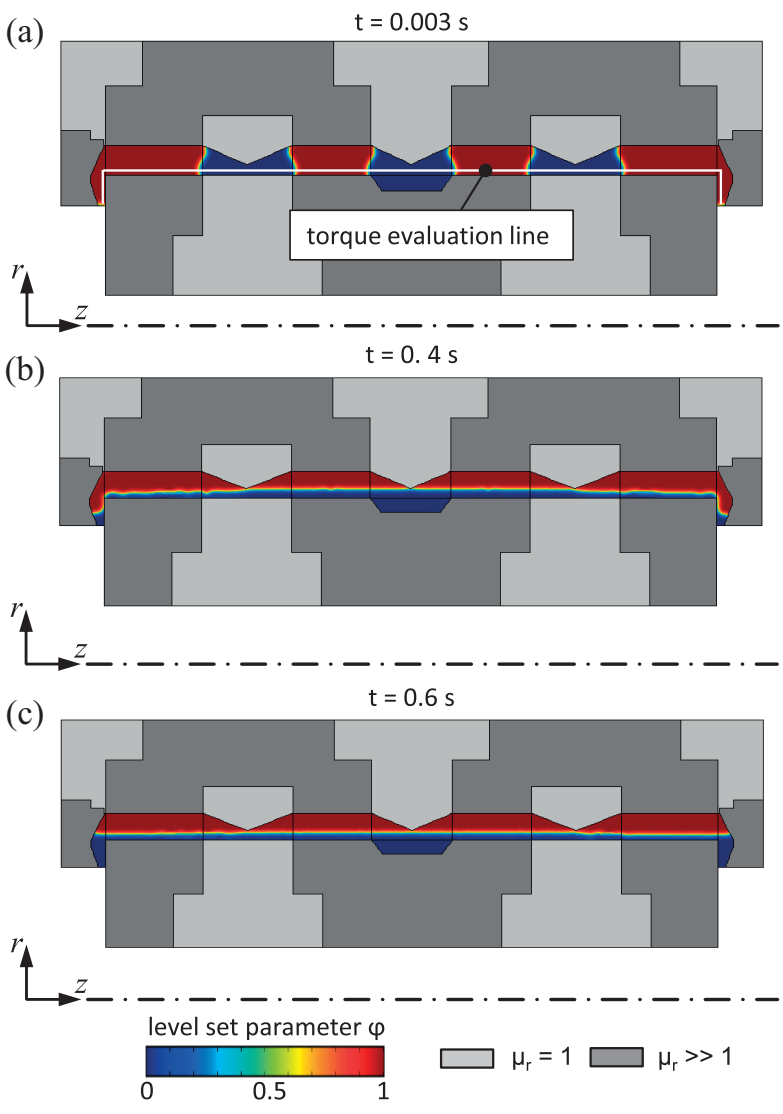

Figure I I. Simulated distribution of the MRF in the shear gap at the rotational speed $\omega=105 \mathrm{~s}^{-1}$ according to Figure 10 .

torque $T$ is performed along a evaluation contour shown in Figure 11

$$
d T=r \cdot\left(\tau_{M}+\tau_{v i s}\right) \cdot \frac{1}{2}(\operatorname{sgn}(\varphi(z-0.5))+1) d A
$$

Under consideration of the sgn function, the level set function $\varphi(z)$ distinguishes between air and MRF along this torque evaluation contour, see Figure 11 showing also the distribution of the MRF for different simulation times.

In contrast to the measurements in Figure 5, the simulated torque can be divided into a magnetically induced torque $T_{M}$ and a viscous-induced torque $T_{v i s}$. The considered rotational speeds for this investigation are $\omega=105 \mathrm{~s}^{-1}$ and $\omega=84 \mathrm{~s}^{-1}$. While the first speed is sufficient to eliminate any drag torques by the MRF, the acceleration forces at $\omega=84 \mathrm{~s}^{-1}$ cannot ensure a drag-free operation. The simulation starts at the time $t=0 \mathrm{~s}$ with an acceleration phase, whereby the MRF is placed in an initial position connecting the inner rotor and outer rotor, compare Figure 11(a). The magnetic flux density in the sealing gap causes a constant magnetic-induced torque of $T_{M}=0.2 \mathrm{~N} \mathrm{~m}$, represented by the green curve in Figure 10. Instead, the viscous-induced torque in the shear and sealing gap 
increases during the acceleration phase, as observed in the previous investigation (Figure 5). Under the impact of the rotational speed, the MRF moves to the outer rotor, and the connection between the inner rotor and outer rotor decreases, Figure 11(b). The decreasing connection of the surface results in a declining simulated torque, compare Figure 10. After a time of $t=0.6 \mathrm{~s}$, the drag torque diminishes completely, and the contact between the inner rotor and outer rotor no longer exists, Figure 11(c). The simulation shows an appropriate interaction between the MRF movement control and the smart sealing. When a reduced rotational speed of $\omega \leq 84 \mathrm{~s}^{-1}$ is applied, a slight torque transmission can occur due to the slight magnetic field and also parts of the MRF remaining in the sealing gap, visualized by the red curve in Figure 10. Thus, the critical speed for this test actuator should be above $\omega_{c}>84 \mathrm{~s}^{-1}$. The critical speed $\omega_{c}$ depends also on the design of the MRF actuator itself, especially on the shear gap diameter. Hence, the critical speed $\omega_{c}$ can be reduced further. On one hand, the critical speed can be reduced by increasing the diameter of the actuator and by the rotational acceleration. On the other hand, the magnetic field of the PM can be compensated by the EM to reduce the magically induced forces.

\section{Experimental investigation}

According to the simulation results, the optimized MRF test actuator was realized. Although the realized MRF actuator is a clutch, the presented experimental investigation of the torque behavior of the MRF sealing in Figure 12 only considers the rotational speed of the outer rotor $\omega_{1}$. This scenario causes the highest

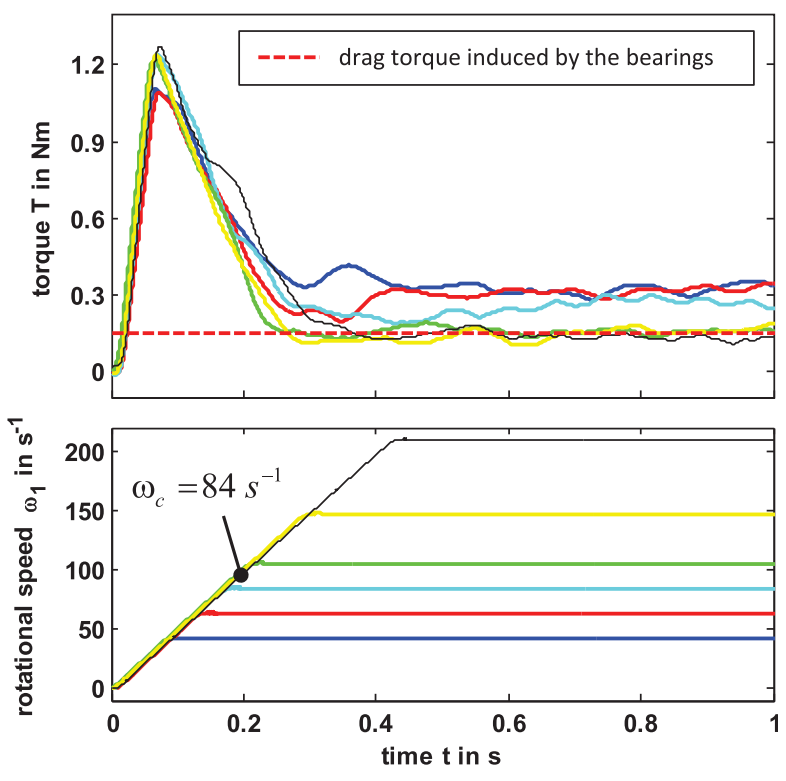

Figure 12. Measurements of the torque behavior of the optimized MRF sealing for different rotational speeds $\omega_{1}$. shear rates and, consequently, the biggest impact on the torque behavior. The following measurements of the drag torque are performed after a certain torque transmission scenario with a operation time of $5 \mathrm{~min}$ and a subsequent downtime of $15 \mathrm{~min}$. During this downtime, the MRF will be moved into the lower section of the sealing gap by gravity. The sedimentation is prevented by the PM as investigated before. The recorded measurements after the mentioned downtime begin with an acceleration phase for certain rotational speeds of $\omega_{1}$, as shown in Figure 12. With increasing speed, the measured torque $T$ rises up to $T=1.23 \mathrm{~N} \mathrm{~m}$. This increase can be explained by the distribution of the MRF over the circumference of the sealing gap and the increasing shear rate, which was already observed in the simulation in Figure 10. Further increasing speeds result in a decrease in the measured torque $T$ due to the MRF leaving the sealing gap. The following decrease in the measured torque is almost comparable within the first interval. Rotational speeds of $\omega_{1}>84 \mathrm{~s}^{-1}$ ensured a drag torque-free operation, except the drag torque of the bearings, which shows a good accordance with the applied analyses before. The mentioned drag torque of the bearings is characterized by the dashed red curve, representing the averaged value. A further improvement can be achieved when during the acceleration phase the magnetic field of the PM is compensated by the EM as mentioned. In contrast to the previous MRF-based clutch with the radial shaft sealings in Figure 1(c), the drag torque is significantly reduced also under consideration of the higher effective radius of the smart sealing. However, the experimental investigation confirms the modeling approach and demonstrates an effective measure to reduce the drag torque of MRFbased actuators to a minimum by utilizing a smart sealing. An exemplary application could be an MRF-based starting clutch in a powertrain of vehicles, resulting in the typical idle mode speed of $\omega=85 \mathrm{~s}^{-1}$. Of course, the lossless operation should be achievable below the idle mode speed to provide the highest energy efficiency. Based on current research, an appropriate design of an MRF-based clutch transmitting a torque of more than $200 \mathrm{~N}$ m results in a sealing gap radius of $r_{s}=130 \mathrm{~mm}$. As already shown in equation (5), the movement of the MRF is based on volume forces. Assuming an identical sealing gap design, an increased sealing gap radius $r_{s}$ will reduce the speed requirements for the lossless operation due to enhanced acceleration forces

$$
\omega_{c, \text { app }}=\sqrt{\frac{r_{s, \exp }}{r_{s, \text { app }}} \cdot \omega_{c, \exp }^{2}}
$$

Therein, the radius of the sealing gap of the experimentally investigated MRF test actuator is $r_{s, \exp }=70 \mathrm{~mm}$, and the radius of the sealing gap of the exemplary MRF-based clutch application is $r_{s, a p p}=130 \mathrm{~mm}$. 
(a)

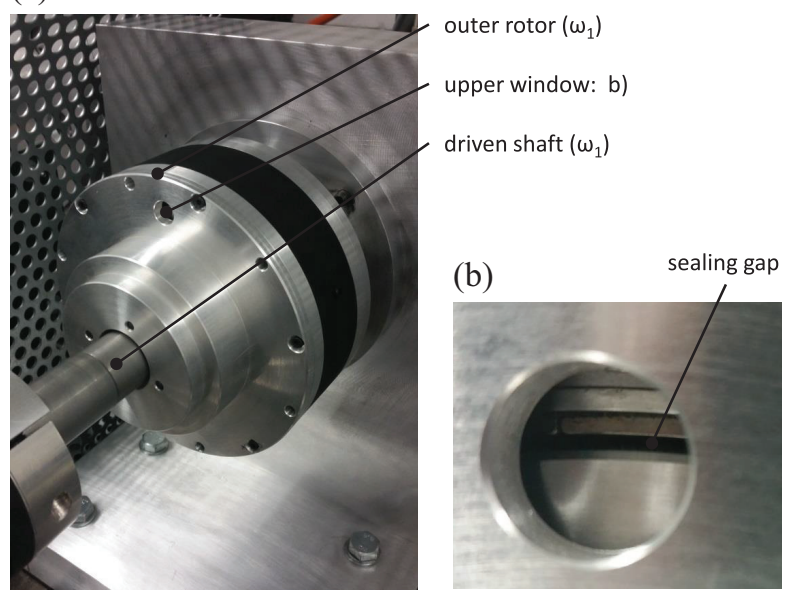

Figure 13. (a) Improved MRF test actuator mounted on the test bench (b) with a zoom of the lower window to observe the tightness.

Consequently, the critical rotational speed can be reduced to $\omega_{c, a p p}<62 \mathrm{~s}^{-1}$, to ensure a lossless operation in idle mode.

\section{Tightness}

After certain dynamical experimental investigations, the tightness of the MRF test actuator was investigated after a standstill of 6 months. During this downtime, the PM provides the calculated magnetic flux density in the shear gap. The tightness was observed through an upper and lower window at the sealing gap of the actuator, see Figure 13. Daily observations confirmed that the carrier liquid did not leak and thus confirmed the modeling results that sedimentation could be avoided.

\section{Conclusion}

In this contribution, measures for a further reduction of the drag torque of MRF-based actuators were developed. While viscous-induced drag torque can be already eliminated by the MRF movement control, the objective of this publication was the minimization of the drag torque caused by the sealings. To overcome both drawbacks, a MRF-based sealing combined with a MRF movement control was proposed and realized as a MRF test actuator to prove both aimed targets. By experimental investigation, a drag torque-free operation was demonstrated in accordance with an analytically calculated critical rotational speed $\omega_{c}$. But due to sedimentation of the MRF particles, a leakage of the carrier liquid occurred during longer downtimes. Therefore, an optimized design of the magnetic sealing was developed, reducing, in addition, the number of PMs for the smart sealing function to only one. Therein, the tightness of the smart sealing was improved by an additional magnetic field of the PM "freezing" the MRF and thus preventing the sedimentation of particles. Since no speraration between fluid and particles occurs, any leakage of carrier liquid during longer downtimes is avoided. For the design of the smart sealing, a model-based approach was presented. The first one is used to determine the minimum flux density to prevent the sedimentation, while by the second one, the drag torque behavior is described in detail. The approach is in good accordance with the experimental investigations and appropriate for future designs of smart sealings using the MRF effect itself.

\section{Declaration of Conflicting Interests}

The authors declared no potential conflicts of interest with respect to the research, authorship, and/or publication of this article.

\section{Funding}

The author(s) disclosed receipt of the following financial support for the research, authorship, and/or publication of this article: This contribution is accomplished within the project PHEVplus, funded by the Federal Ministry for Economic Affairs and Energy (BMWi) of Germany under grant number 01MY13004B.

\section{ORCID iD}

Christian Hegger (iD) https://orcid.org/0000-0003-1046-4231

\section{References}

Borbath T, Bica D, Potencz I, et al. (2010) Magnetic nanofluids and magnetic composite fluids in rotating seal systems. IAHR Symposium on Hydraulic Machinery and Systems 12: 012105.

Borbath T, Bica D, Potencz I, et al. (2011) Leakage-free rotating seal systems with magnetic nanofluids and magnetic composite fluids designed for various applications. International Journal of Fluid Machinery and Systems 4: 67-75.

Carlson J (2007) Chapter 6.6: magnetorheological fluid actuators. In: Janocha H (ed.) Adaptronics and Smart Structures: Basics, Materials, Design, and Applications. 2nd ed. Berlin: Springer, pp. 184-204.

Coulter J, Weiss K and Carlson J (1993) Engineering applications of electro-rheological materials. Journal of Intelligent Material Systems and Structures 4: 248-259.

Eagle Industry Co., Ltd (2014) Magnetic-fluid-seal-CAT. Available at: www.ekkeagle.com

Fujita D, Yoshimura K, Seki Y, et al. (1999) Characterization of magnetorheological suspensions for seal. Journal of Intelligent Material Systems and Structures 10: 770-774.

Gratzer F, Steinwender H and Kusej A (2008) Magnetorheological four wheel clutch. ATZ 10: 902-909.

Güth D and Maas J (2015) MRF-brake for use in wind turbines. In: Proceedings of the SMASIS, Colorado Springs, $\mathrm{CO}$, September.

Güth D and Maas J (2016) Characterization and modeling of the behavior of magnetorheological fluids at high shear 
rates in rotational systems. Journal of Intelligent Material Systems and Structures 27: 5689-5704.

Güth D, Schamoni M and Maas J (2013) Magnetic fluid control for viscous loss reduction of high-speed MRF brakes and clutches with well-defined fail-safe behavior. Smart Materials and Structures 22: 094010.

Hegger C and Maas J (2016a) Design and evaluation of energy-efficient MRF-based coupling elements for a multimode transmission. In: Proceedings of the FISITA world automotive congress, 26-30 September 2016, Busan, Korea.

Hegger C and Maas J (2016b) Investigation of energy-efficient MRF-based clutches for hybrid power trains. SPIE Active and Passive Smart Structures and Integrated Systems. Available at: https://www.spiedigitallibrary.org/conferenceproceedings-of-spie/9799/97992W/Investigation-of-energyefficient-MRF-based-clutches-for-hybrid-powertrains/10.11 17/12.2219689.short?SSO = 1

Herber S, Horst J, Gassmann T, et al. (2016) "PHEVplus": efficient Plug-in-hybrid System with MRF-coupling Technology. In: Proceedings of the FISITA world automotive congress, 26-30 September 2016, Busan, Korea.

Kordonski W and Gorodkin S (1996) Magnetorheological fluid-based seal. Journal of Intelligent Materials Systems and Structures 7: 569-572.

Lampe D (2000) Untersuchungen Zum Einsatz Von Magnetorheologischen Fluiden in Kupplungen. PhD Thesis, Technical University Dresden, Dresden.

Lampe D and Thess A (1998) MRF-clutch: design considerations and performance. In: Proceedings of the actuator 1998, 6th international conference on new actuators, Bremen, 17-19 June, pp. 449-452. Available at: http:// www.donnerflug.de/publikationen/ Lampe_Actuator1998_Bremen_p81.pdf
Maas J, Güth D and Wiehe A (2011) MRF-actuator concepts for HMI and industrial applications. SPIE Smart Structures. Available at: https://www.spiedigitallibrary.org/con ference-proceedings-of-spie/7977/797714/MRF-actuatorconcepts-for-HMI-and-industrial-applications/10.1117/12. 886376.short

Nakano M (2016) Development of MR fluid brake for a super-compact electric vehicle. In: Proceedings of the Plenary Lecture 09 at the ERMR, 04-09 July, Incheon, Korea.

Potoczny M and Zachara B (2012) Influence of magnetorheological fluid volume onto obtained critical pressures on rotary shaft seals. Key Engineering Materials 490: 119-127.

Raj K and Moskowitz R (1990) Commercial applications of ferrofluids. Journal of Magnetism and Magnetic Materials 85: 233-245.

Silvestri M, Prati E and Tasora A (2006) Radial lip seals efficiency under dynamic operating conditions. International Conference on Tribology. Available at: http://projectchro no.org/tasora/pubblicazioni/article-aitc-tenute.pdf

Smith A, Ulicny J and Kennedy L (2007) Magnetorheological fluid fan drive for trucks. Journal of Intelligent Material Systems and Structures 18: 1131-1136.

Vicente J, Delgado AV, Plaza RC, et al. (2000) Stability of cobalt ferrite colloidal particles: effect of $\mathrm{pH}$ and applied magnetic fields. American Chemical Society, Langmuir 16: 7954-7961.

Xiang C, Zhang Y and Liu H (2013) Research on drag torque of high relative speed open wet clutch of vehicle. Chinese Journal of Mechanical Engineering 49: 71-77.

Zhao S, Sheng Q, Lin S, et al. (2016) The model of seal mechanism for magnetic fluid and related experimental study. MECHANIKA 22: 260-264. 